\title{
On the lower bound of the discrepancy of Halton's sequence II
}

\author{
Mordechay B. Levin ${ }^{1}$
}

Received: 30 July 2015 / Revised: 1 March 2016 / Accepted: 3 March 2016 /

Published online: 31 March 2016

(C) Springer International Publishing AG 2016

Abstract Let $\left(H_{S}(n)\right)_{n \geqslant 1}$ be an $s$-dimensional generalized Halton's sequence. Let $D_{N}^{*}$ be the discrepancy of the sequence $\left(H_{s}(n)\right)_{n=1}^{N}$. It is known that $N D_{N}^{*}=\mathrm{O}\left(\ln ^{s} N\right)$ as $N \rightarrow \infty$. In this paper, we prove that this estimate is exact. Namely, there exists a constant $C\left(H_{S}\right)>0$ such thats

$$
\max _{1 \leqslant M \leqslant N} M D_{M}^{*} \geqslant C\left(H_{S}\right) \log _{2}^{s} N \quad \text { for } \quad N=2,3, \ldots
$$

Keywords Halton's sequence · Ergodic adding machine

Mathematics Subject Classification $11 \mathrm{~K} 38$

\section{Introduction}

Let $\left(\beta_{n}\right)_{n \geqslant 1}$ be a sequence in the unit cube $[0,1)^{s}, B_{\mathbf{y}}=\left[0, y_{1}\right) \times \cdots \times\left[0, y_{s}\right)$,

$$
\Delta\left(B_{\mathbf{y}},\left(\beta_{n}\right)_{n=1}^{N}\right)=\sum_{n=1}^{N}\left(\mathbf{1}_{B_{\mathbf{y}}}\left(\beta_{n}\right)-y_{1} \cdots y_{s}\right),
$$

where $\mathbf{1}_{B_{\mathbf{y}}}(\mathbf{x})=1$ if $\mathbf{x} \in B_{\mathbf{y}}$, and $\mathbf{1}_{B_{\mathbf{y}}}(\mathbf{x})=0$ if $\mathbf{x} \notin B_{\mathbf{y}}$.

$\triangle$ Mordechay B. Levin

mlevin@math.biu.ac.il

1 Department of Mathematics, Bar-Ilan University, Ramat-Gan 52900, Israel 
We define the star discrepancy of an $N$-point set $\left(\beta_{n}\right)_{n=1}^{N}$ as

$$
D^{*}\left(\left(\beta_{n}\right)_{n=1}^{N}\right)=\sup _{0<y_{1}, \ldots, y_{s} \leqslant 1}\left|\frac{\Delta\left(B_{\mathbf{y}},\left(\beta_{n}\right)_{n=1}^{N}\right)}{N}\right|
$$

In $1954, \mathrm{~K}$. Roth proved that

$$
\limsup _{N \rightarrow \infty} N(\ln N)^{-s / 2} D^{*}\left(\left(\beta_{n}\right)_{n=1}^{N}\right)>0
$$

According to the well-known conjecture (see, e.g., [1, p. 283]), this estimate can be improved to

$$
\limsup _{N \rightarrow \infty} N(\ln N)^{-s} D^{*}\left(\left(\beta_{n}\right)_{n=1}^{N}\right)>0
$$

In 1972, W. Schmidt proved this conjecture for $s=1$. For $s=2$, Faure and Chaix [4] proved (3) for a class of $(t, s)$-sequences. See [2] for the most important results on this conjecture.

There exists another conjecture on the lower bound for the discrepancy function: there exists a constant $\dot{c}_{3}>0$ such that

$$
N D^{*}\left(\left(\beta_{k, N}\right)_{k=0}^{N-1}\right)>\dot{c}_{3}(\ln N)^{s / 2}
$$

for all $N$-point sets $\left(\beta_{k, N}\right)_{k=0}^{N-1}$ (see [2, p. 147]).

Definition An $s$-dimensional sequence $\left(\left(\beta_{n}\right)_{n} \geqslant 1\right)$ is of low discrepancy (abbreviated 1.d.s.) if $D^{*}\left(\left(\beta_{n}\right)_{n=1}^{N}\right)=\mathrm{O}\left(N^{-1}(\ln N)^{s}\right)$ for $N \rightarrow \infty$.

Let $p \geqslant 2$ be an integer,

$$
n=\sum_{j \geqslant 1} e_{p, j}(n) p^{j-1}, \quad e_{p, j}(n) \in\{0,1, \ldots, p-1\}, \quad \phi_{p}(n)=\sum_{j \geqslant 1} e_{p, j}(n) p^{-j}
$$

van der Corput proved that $\left(\phi_{p}(n)\right)_{n \geqslant 0}$ is a 1-dimensional 1.d.s. (see [12]). Let

$$
\widehat{H}_{S}(n)=\left(\phi_{\hat{p}_{1}}(n), \ldots, \phi_{\hat{p}_{s}}(n)\right), \quad n=0,1,2, \ldots,
$$

where $\widehat{p}_{1}, \ldots, \widehat{p}_{S} \geqslant 2$ are pairwise coprime integers. Halton proved that $\left(\widehat{H}_{S}(n)\right)_{n \geqslant 0}$ is an $s$-dimensional 1.d.s. (see [6]). For other examples of 1.d.s. see, e.g., $[1,5,11]$. In [9], we proved that Halton's sequence satisfies (3). In this paper we generalize this result.

Let $Q=\left(q_{1}, q_{2}, \ldots\right)$ and $Q_{j}=q_{1} q_{2} \cdots q_{j}$, where $q_{j} \geqslant 2, j=1,2, \ldots$, is a sequence of integers. Consider Cantor's expansion of $x \in[0,1)$ :

$$
x=\sum_{j=1}^{\infty} \frac{x_{j}}{Q_{j}}, \quad x_{j} \in\left\{0,1, \ldots, q_{j}-1\right\}, \quad x_{j} \neq q_{j}-1 \text { for infinitely many } j .
$$


The $Q$-adic representation of $x$ is then unique. We define the odometer transform as

$$
T_{Q}(x)=\frac{x_{k}+1}{Q_{k}}+\sum_{j \geqslant k+1} \frac{x_{j}}{Q_{j}}, \quad T_{Q}^{n}(x)=T_{Q}\left(T_{Q}^{n-1}(x)\right),
$$

$n=2,3, \ldots, T_{Q}^{0}(x)=x$, where $k=\min \left\{j: x_{j} \neq q_{i}-1\right\}$.

For $Q=(q, q, \ldots)$, we obtain von Neumann-Kakutani's $q$-adic adding machine (see, e.g., [5]). As is known, the sequence $\left(T_{Q}^{n}(x)\right)_{n \geq 1}$ coincides for $x=0$ with the van der Corput sequence (see, e.g., [5, Section 2.5]).

Let $q_{0} \geqslant 4, p_{i, j} \geqslant 2, s \geqslant i \geqslant 1, j \geqslant 1$, be integers, g.c.d. $\left(p_{i, k}, p_{j, l}\right)=1$ for $i \neq j, \mathcal{P}_{i}=\left(p_{i, 1}, p_{i, 2}, \ldots\right), \mathcal{P}=\left(\mathcal{P}_{1}, \ldots, \mathcal{P}_{s}\right), T_{\mathcal{P}}(\mathbf{x})=\left(T_{\mathcal{P}_{1}}\left(x_{1}\right), \ldots, T_{\mathcal{P}_{s}}\left(x_{s}\right)\right)$,

$$
\begin{aligned}
& \widetilde{P}_{i, 0}=1, \quad \widetilde{P}_{i, j}=\prod_{1 \leqslant k \leqslant j} p_{i, k}, \quad \widetilde{P}_{i, j} \leqslant q_{0}^{j / 2}, \quad i \in[1, s], \quad j \geqslant 1, \\
& n=\sum_{j \geqslant 1} e_{p_{i, j}, j}(n) \widetilde{P}_{i, j-1}, \quad e_{p_{i, j}, j}(n) \in\left\{0,1, \ldots, p_{i, j}-1\right\}, \quad n=0,1, \ldots, \\
& \varphi_{\mathcal{P}_{i}}(n)=\sum_{j \geqslant 1} e_{p_{i, j}, j}(n) \widetilde{P}_{i, j}^{-1}, \quad H_{\mathcal{P}}(n)=\left(\varphi_{\mathcal{P}_{1}}(n), \ldots, \varphi \mathcal{P}_{s}(n)\right) .
\end{aligned}
$$

We note that $H_{\mathcal{P}}(n)=T_{\mathcal{P}}^{n}(\mathbf{0})$ for $n=0,1, \ldots$

Let $\Sigma_{i}=\left(\sigma_{i, j}\right)_{j \geqslant 1}$ be a sequence of corresponding permutations $\sigma_{i, j}$ of $\{0,1, \ldots$, $\left.p_{i, j}-1\right\}$ for $j \geqslant 1, \Sigma=\left(\Sigma_{1}, \ldots, \Sigma_{s}\right), \mathbf{x}=\left(x_{1}, \ldots, x_{s}\right)$,

$$
\widetilde{\Sigma}(\mathbf{x})=\left(\widetilde{\Sigma}_{1}\left(x_{1}\right), \ldots, \widetilde{\Sigma}_{s}\left(x_{s}\right)\right), \quad \widetilde{\Sigma}_{i}\left(x_{i}\right)=\sum_{j \geqslant 1} \frac{\sigma_{i, j}\left(x_{i, j}\right)}{\widetilde{P}_{i, j}}, \quad x_{i}=\sum_{j \geqslant 1} \frac{x_{i, j}}{\widetilde{P}_{i, j}} .
$$

We consider the following generalization of Halton's sequence (see $[3,5,7])$ :

$$
H_{\mathcal{P}}^{\Sigma}(n, \mathbf{x})=\widetilde{\Sigma}\left(T_{\mathcal{P}}^{n}(\mathbf{x})\right), \quad n=0,1,2, \ldots
$$

We note that $\left(H_{\mathcal{P}}^{\Sigma}(n, \mathbf{x})\right)_{n \geqslant 0}$ coincides for $\mathbf{x}=\mathbf{0}$ and $s=1$ with the Faure sequence $S_{Q}^{\Sigma}$ [3]. Similarly to [11, pp. 29-31], we get that $\left(H_{\mathcal{P}}^{\Sigma}(n, \mathbf{x})\right)_{n \geqslant 0}$ is of low discrepancy.

\section{Theorem and its proof}

In this section we will prove

Theorem Let $s \geqslant 2, C_{1}=s q_{0}^{s+1} \log _{2} q_{0}, C=8 q_{0}^{s} C_{1}^{s}$ and $\log _{2} N \geqslant 2 q_{0}^{s} C_{1}$. Then

$$
\inf _{\mathbf{x} \in[0,1)^{s}} \max _{1 \leqslant M \leqslant N} M D^{*}\left(\left(H_{\mathcal{P}}^{\mathbf{\Sigma}}(n, \mathbf{x})\right)_{n=1}^{M}\right) \geqslant C^{-1} \log _{2}^{s} N
$$

This result supports conjecture (3) (see also $[8,10]$ ). 
The proof of Theorem is similar to the proof of [9, Theorem]. The main part of the proof in [9] and in this paper is the construction of the bounded vector $\left(y_{1}, \ldots, y_{s}\right)$ and the application of the Chinese Remainder Theorem. In the paper [9], we take $y_{i}=\sum_{j=1}^{m} p_{i}^{-\tau_{i, j}}, i=1, \ldots, s$, where

$$
\tau_{i, j}=\tau_{i} j, \quad j=1,2, \ldots, \quad p_{i}^{\tau_{i}} \equiv 1\left(\bmod \frac{p_{1} \cdots p_{s}}{p_{i}}\right), \quad \tau_{i} \in\left[1, p_{1} \cdots p_{s}\right]
$$

In this paper we take $y_{i}=\sum_{j=1}^{m} \widetilde{P}_{i, \tau_{i, j}}^{-1}$, with some special sequences $\left(\tau_{i, j}\right)_{1 \leqslant i \leqslant s, j \geqslant 1}$. In order to obtain the 'periodic' properties similar to (8), we need a more complicated construction of $\left(\tau_{i, j}\right)_{s} \geqslant i \geqslant 1, j \geqslant 1$ :

- $p_{i, \tau_{i, j}}=p_{i, \tau_{i, 1}}, j=1,2, \ldots$,

- $\sigma_{i, \tau_{i, j}}^{-1}(0)-\sigma_{i, \tau_{i, j}}^{-1}(1) \equiv \sigma_{i, \tau_{i, 1}}^{-1}(0)-\sigma_{i, \tau_{i, 1}}^{-1}(1)\left(\bmod p_{i, \tau_{i, 1}}\right), j=1,2, \ldots$,

- $\widetilde{P}_{i, \tau_{i, j}} \equiv \widetilde{P}_{i, \tau_{i, 1}}\left(\bmod p_{1} \cdots p_{s} / p_{i}\right), j=1,2, \ldots$,

in such a way that the sets $\left\{\tau_{i, 1}, \tau_{i, 2}, \ldots\right\} \cap[1, \mathfrak{m}]$ would receive the greatest length, where $\mathfrak{m}=\left[2 s^{-1} \log _{q_{0}} N\right], s \geqslant i \geqslant 1$. We need all these conditions to prove statement (26).

In order to construct $\left(\tau_{i, j}\right)_{1 \leqslant i \leqslant s, j} \geqslant 1$, we define auxiliary sequences $\mathcal{L}_{i, j}^{(\mathfrak{m})}, L_{i}^{(\mathfrak{m})}$, $l_{i, j}, \mathcal{F}_{i, b}^{(\mathfrak{m})}, \ldots$

\subsection{Construction of the sequence $\left(\tau_{i, j}\right)$}

Let $\mathfrak{m}=\left[2 s^{-1} \log _{q_{0}} N\right], \mathfrak{L}_{i}^{(\mathfrak{m})}=\left\{1 \leqslant k \leqslant \mathfrak{m}: p_{i, k} \leqslant q_{0}\right\}$. By (5), we get

$$
q_{0}^{\mathfrak{m} / 2} \geqslant \prod_{j \in[1, \mathfrak{m}] \backslash \mathfrak{L}_{i}^{(\mathfrak{m})}} p_{i, j}>q_{0}^{\mathfrak{m}-\# \mathfrak{L}_{i}^{(\mathfrak{m})}}
$$

Hence

$$
\# \mathfrak{L}_{i}^{(\mathfrak{m})}>\frac{\mathfrak{m}}{2}
$$

Let $a_{i, j} \equiv \sigma_{i, j}^{-1}(0)-\sigma_{i, j}^{-1}(1)\left(\bmod p_{i, j}\right), a_{i, j} \in\left\{1, \ldots, p_{i, j}-1\right\}, \mathfrak{a} \in\left\{1, \ldots, q_{0}\right\}$,

$$
\begin{aligned}
\mathcal{L}_{i, j, \mathfrak{a}}^{(\mathfrak{m})} & =\left\{k \in \mathfrak{L}_{i}^{(\mathfrak{m})}: p_{i, k}=p_{i, j}, a_{i, k}=\mathfrak{a}\right\}, \\
L_{i}^{(\mathfrak{m})} & =\max _{1 \leqslant j \leqslant \mathfrak{m}, 1 \leqslant \mathfrak{a} \leqslant q_{0}} \# \mathcal{L}_{i, j, \mathfrak{a}}^{(\mathfrak{m})}, \quad 1 \leqslant i \leqslant s .
\end{aligned}
$$

It is easy to see that there exist $g_{i, \mathfrak{m}} \in[1, \mathfrak{m}]$ and $\mathfrak{a}_{i}=\mathfrak{a}_{i, m} \in\left[1, q_{0}\right]$ such that

$$
\# \mathcal{L}_{i, g_{i, \mathfrak{m}}, \mathfrak{a}_{i}}^{(\mathfrak{m})}=L_{i}^{(\mathfrak{m})}, \quad 1 \leqslant i \leqslant s .
$$


We enumerate the set $\mathcal{L}_{i, g_{i, \mathfrak{m}}, \mathfrak{a}_{i}}^{(\mathfrak{m})}$ :

$$
\mathcal{L}_{i, g_{i, \mathfrak{m}}, \mathfrak{a}_{i}}^{(\mathfrak{m})}=\left\{l_{i, 1}<\cdots<l_{i, L_{i}^{(\mathfrak{m})}}\right\}
$$

For $i \in[1, s]$ we have

$$
L_{i}^{(\mathfrak{m})} \geqslant \frac{\# \mathfrak{L}_{i}^{(\mathfrak{m})}}{q_{0}^{2}} \geqslant \frac{\mathfrak{m}}{2 q_{0}^{2}}, \quad l_{i, L_{i}^{(\mathfrak{m})}} \leqslant \mathfrak{m}, \quad a_{i, l_{i, j}}=\mathfrak{a}_{i}, \quad j \in\left[1, L_{i}^{(\mathfrak{m})}\right] .
$$

Let $p_{i}=p_{i}^{(\mathfrak{m})}=p_{i, g_{i, \mathfrak{m}}} \leqslant q_{0}, p_{0}=p_{0}^{(\mathfrak{m})}=p_{1} p_{2} \cdots p_{s} \leqslant q_{0}^{s}, \dot{p}_{i}=p_{0} / p_{i} \leqslant q_{0}^{s-1}$ and

$$
\mathcal{F}_{i, b}^{(\mathfrak{m})}=\left\{1 \leqslant k \leqslant L_{i}^{(\mathfrak{m})}: \widetilde{P}_{i, l_{i, k}}^{-1} \equiv b\left(\bmod \dot{p}_{i}\right)\right\}
$$

We define $F_{i}, m$ and $b_{i}=b_{i}^{(\mathfrak{m})}$ as follows:

$$
F_{i}=F_{i}^{(\mathfrak{m})}=\# \mathcal{F}_{i, b_{i}}^{(\mathfrak{m})}=\max _{0 \leqslant b<\dot{p}_{i}} \# \mathcal{F}_{i, b}^{(\mathfrak{m})}, \quad m=\min _{1 \leqslant i \leqslant s} F_{i}^{(\mathfrak{m})}
$$

We enumerate the set $F_{i, b_{i}}^{(\mathfrak{m})}$ :

$$
\mathcal{F}_{i, b_{i}}^{(\mathfrak{m})}=\left\{f_{i, 1}<\cdots<f_{i, F_{i}}\right\}, \quad \mathfrak{m}=\left[2 s^{-1} \log _{q_{0}} N\right] .
$$

Bearing in mind that $\log _{2} N \geqslant 2 q_{0}^{s} C_{1}$ and $C_{1}=s q_{0}^{s+1} \log _{2} q_{0}$, we have

$$
m \geqslant \min _{1 \leqslant i \leqslant s} \frac{L_{i}^{(\mathfrak{m})}}{\dot{p}_{i}} \geqslant \min _{1 \leqslant i \leqslant s} \frac{\mathfrak{m}}{2 q_{0}^{2} \dot{p}_{i}} \geqslant \frac{\mathfrak{m} q_{0}^{-s-1}}{2} \geqslant C_{1}^{-1} \log _{2} N \geqslant 2 q_{0}^{s} \geqslant 2 p_{0} .
$$

Let $\mathbf{k}=\left(k_{1}, \ldots, k_{s}\right), \tau_{i, j}=l_{i, f_{i, j}}, \boldsymbol{\tau}_{\mathbf{k}}=\left(\tau_{1, k_{1}}, \ldots, \tau_{s, k_{s}}\right), P_{i, k}=\widetilde{P}_{i, \tau_{i, k}}$,

$$
P_{\mathbf{k}}=\prod_{i=1}^{s} P_{i, k_{i}}, \quad M_{i, \mathbf{k}}=\widetilde{M}_{i, \tau_{\mathbf{k}}}, \quad \text { with } \quad \widetilde{M}_{i, \mathbf{k}} \equiv \prod_{\substack{1 \leqslant j \leqslant s \\ j \neq i}} \widetilde{P}_{j, k_{j}}^{-1}\left(\bmod \widetilde{P}_{i, k_{i}}\right) .
$$

Applying (10), we get $\tau_{i, m}=l_{i, f_{i, m}} \leqslant l_{i, F_{i}^{(\mathfrak{m})}} \leqslant l_{i, L_{i}^{(\mathfrak{m})}} \leqslant \mathfrak{m}$. Let $\mathbf{m}=(m, \ldots, m)$. From (5) and (14), we derive

$$
2 P_{\mathbf{m}} \leqslant 2 \prod_{i=1}^{s} \widetilde{P}_{i, \tau_{i, k}} \leqslant 2 q_{0}^{s \mathfrak{m} / 2}=q_{0}^{s\left[2 s^{-1} \log _{q_{0}} N\right] / 2} \leqslant N .
$$

We will need the following properties of integers $\mathfrak{a}_{i}, 1 \leqslant i \leqslant s$, (see (16), (17)): By (11), we have that $\left(b_{i}, \dot{p}_{i}\right)=1$ and $\left(b_{j}, p_{i}\right)=1$ for $i \neq j, i, j=1, \ldots, s$. Let 
$c_{i} \equiv \prod_{1 \leqslant j \leqslant s, j \neq i} b_{j}\left(\bmod p_{i}\right)$. According to $(10),(11)$ and $(14)$, we obtain

$$
\left(c_{i}, p_{i}\right)=1, \quad M_{i, \mathbf{k}} \equiv c_{i}\left(\bmod p_{i}\right), \quad a_{i, \tau_{i, j}}=\mathfrak{a}_{i}, \quad j \geqslant 1, \quad i \in[1, s] .
$$

Let

$$
\widetilde{p}_{i}=\text { g.c.d. }\left(\mathfrak{a}_{i}, p_{i}\right), \quad \widehat{p}_{i}=\frac{p_{i}}{\widetilde{p}_{i}}, \quad \widehat{a}_{i}=\frac{\mathfrak{a}_{i}}{\widetilde{p}_{i}}, \quad d_{i} \equiv c_{i} \mathfrak{a}_{i}\left(\bmod \widehat{p}_{i}\right),
$$

$d_{i} \in\left\{1, \ldots, \widehat{p}_{i}-1\right\}$. Hence

$$
\frac{d_{i}}{\widehat{p}_{i}} \equiv c_{i} \frac{\mathfrak{a}_{i}}{p_{i}}(\bmod 1), \quad\left(d_{i}, \widehat{p}_{i}\right)=1, \quad \widehat{p}_{i}>1, \quad i=1, \ldots, s .
$$

\subsection{Using the Chinese Remainder Theorem}

Let $x_{i}=\sum_{j \geqslant 1} x_{i, j} \widetilde{P}_{i, j}^{-1}$, with $x_{i, j} \in\left\{0,1, \ldots, p_{i, j}-1\right\}, i=1, \ldots, s$. We define the truncation

$$
\left[x_{i}\right]_{r}=\sum_{1 \leqslant j \leqslant r} x_{i, j} \widetilde{P}_{i, j}^{-1}, \quad r \geqslant 1 .
$$

If $x=\left(x_{1}, \ldots, x_{s}\right) \in[0,1)^{s}$, then the truncation $[\mathbf{x}]_{\mathbf{r}}$ is defined coordinatewise, that is, $[\mathbf{x}]_{\mathbf{r}}=\left(\left[x_{1}\right]_{r_{1}}, \ldots,\left[x_{s}\right]_{r_{s}}\right)$, where $\mathbf{r}=\left(r_{1}, \ldots, r_{s}\right)$.

By (6), we have

$$
\left[\varphi_{\mathcal{P}_{i}}(n)\right]_{r_{i}}=\left[x_{i}\right]_{r_{i}} \Longleftrightarrow n \equiv \sum_{1 \leqslant j \leqslant r} x_{i, j} \widetilde{P}_{i, j-1}\left(\bmod \widetilde{P}_{i, r}\right)
$$

Applying (14) and the Chinese Remainder Theorem, we get

$$
\begin{aligned}
{\left[H_{\mathcal{P}}(n)\right]_{\mathbf{r}} } & =[\mathbf{x}]_{\mathbf{r}} \quad \Longleftrightarrow n \equiv \check{x}_{\mathbf{r}}\left(\bmod \widetilde{P}_{\mathbf{r}}\right), \\
\check{x}_{\mathbf{r}} & \equiv \sum_{i=1}^{s} \widetilde{M}_{i, \mathbf{r}} \widetilde{P}_{\mathbf{r}} \widetilde{P}_{i, r_{i}}^{-1} \sum_{1 \leqslant j \leqslant r} x_{i, j} \widetilde{P}_{i, j-1}\left(\bmod \widetilde{P}_{\mathbf{r}}\right), \quad \check{x}_{\mathbf{r}} \in\left[0, \widetilde{P}_{\mathbf{r}}\right)
\end{aligned}
$$

Now we will find the relation between $T_{\mathcal{P}}^{n}(\mathbf{x})$ and $H_{\mathcal{P}}(n)$ (see (20). It is easy to verify that if $r_{i}^{\prime} \geqslant r_{i}, i=1, \ldots, s$, then $\check{x}_{\mathbf{r}^{\prime}} \equiv \check{x}_{\mathbf{r}}\left(\bmod \widetilde{P}_{\mathbf{r}}\right)$. According to (4), we get

$$
\text { if }[\mathbf{w}]_{\mathbf{r}}=[\mathbf{x}]_{\mathbf{r}}, \quad \text { then }\left[T_{\mathcal{P}}^{n}(\mathbf{w})\right]_{\mathbf{r}}=\left[T_{\mathcal{P}}^{n}(\mathbf{x})\right]_{\mathbf{r}}, \quad n=0,1, \ldots
$$

From (4), (6) and (18), we obtain

$$
\left[T_{\mathcal{P}}^{W}(\mathbf{0})\right]_{\mathbf{r}}=\left[H_{\mathcal{P}}(W)\right]_{\mathbf{r}}=[\mathbf{x}]_{\mathbf{r}}, \quad W=\check{x}_{\mathbf{r}}
$$


Hence

$$
\left[T_{\mathcal{P}}^{n}(\mathbf{x})\right]_{\mathbf{r}}=\left[T_{\mathcal{P}}^{n}\left(T_{\mathcal{P}}^{W}(\mathbf{0})\right)\right]_{\mathbf{r}}=\left[T_{\mathcal{P}}^{n+W}(\mathbf{0})\right]_{\mathbf{r}}=\left[H_{S}(n+W)\right]_{\mathbf{r}}
$$

Let $W_{\mathbf{m}}(\mathbf{x})=\check{x}_{\mathbf{m}} \in\left[0, P_{\mathbf{m}}\right)$. Therefore

$$
\left[T_{\mathcal{P}}^{n}(\mathbf{x})\right]_{\mathbf{r}}=\left[H_{\mathcal{P}}\left(n+W_{\mathbf{m}}(\mathbf{x})\right)\right]_{\mathbf{r}}, \quad 1 \leqslant r_{i} \leqslant m, \quad 1 \leqslant i \leqslant s, \quad n \geqslant 0 .
$$

\subsection{Construction of boundary points $y_{1}, \ldots, y_{s}$ and $u_{1}, \ldots, u_{s}$}

Let $\mathbf{y}=\left(y_{1}, \ldots, y_{s}\right)$ with $y_{i}=\sum_{1 \leqslant j \leqslant m} P_{i, j}^{-1}$, and let $\ddot{y}_{i, k_{i}}=\sum_{1 \leqslant j \leqslant k_{i}} P_{i, j}^{-1}, k_{i} \geqslant 1$, $i=1, \ldots, s, \mathbf{k}=\left(k_{1}, \ldots, k_{s}\right)$,

$$
B_{\mathbf{y}}=\left[0, y_{1}\right) \times \cdots \times\left[0, y_{s}\right), \quad B^{(\mathbf{k})}=\prod_{i=1}^{s}\left[\ddot{y}_{i, k_{i}}-P_{i, k_{i}}^{-1}, \ddot{y}_{i, k_{i}}\right) .
$$

We deduce

$$
B_{\mathbf{y}}=\bigcup_{k_{1}, \ldots, k_{s}=1}^{m} B^{(\mathbf{k})}, \quad \mathbf{1}_{B_{\mathbf{y}}}(\mathbf{z})-y_{1} \cdots y_{s}=\sum_{k_{1}, \ldots, k_{s}=1}^{m}\left(\mathbf{1}_{B^{(\mathbf{k})}}(\mathbf{z})-P_{\mathbf{k}}^{-1}\right)
$$

Consider the following condition:

$$
H_{\mathcal{P}}^{\Sigma}(n, \mathbf{x}) \in B^{(\mathbf{k})} .
$$

In order to express this condition in terms of the sequence $\left(H_{\mathcal{P}}(n)\right)_{n \geqslant 1}$, we will construct boundary points $u_{1}, \ldots, u_{s}$. Next we will construct auxiliary sequences $\mathbf{u}^{(\mathbf{k})}, \breve{u}^{(\mathbf{k})}, A_{\mathbf{k}}, \ldots$ Applying (18), we will get in (26) the solution of (23).

$$
\begin{aligned}
\text { Let } \mathbf{u} & =\left(u_{1}, \ldots, u_{s}\right), u_{i}=\sum_{j \geqslant 1}^{\tau_{i, m}} u_{i, j} \widetilde{P}_{i, j}^{-1} \text { with } u_{i, j}=\sigma_{i, j}^{-1}\left(y_{i, j}\right), u_{i, j}^{*}=\sigma_{i, j}^{-1}(0), \\
\mathbf{u}^{(\mathbf{k})} & =\left(u_{1}^{\left(k_{1}\right)}, \ldots, u_{s}^{\left(k_{s}\right)}\right), \quad u_{i}^{\left(k_{i}\right)}=\sum_{j=1}^{\tau_{i, k_{i}}-1} u_{i, j} \widetilde{P}_{i, j}^{-1}+u_{i, \tau_{i, k_{i}}}^{*} \widetilde{P}_{\tau_{i, k_{i}}}^{-1}, \\
\check{u}^{(\mathbf{k})} & \equiv \sum_{i=1}^{s} M_{i, \mathbf{k}} P_{\mathbf{k}} P_{i, k_{i}}^{-1}\left(\sum_{j=1}^{\tau_{i, k_{i}}-1} u_{i, j} \widetilde{P}_{i, j-1}+u_{i, \tau_{i, k_{i}}^{*}} \widetilde{P}_{i, \tau_{i, k_{i}}-1}\right)\left(\bmod P_{\mathbf{k}}\right), \\
\check{u}_{\mathbf{k}} & \equiv \sum_{i=1}^{s} M_{i, \mathbf{k}} P_{\mathbf{k}} P_{i, k_{i}}^{-1} \sum_{j=1}^{\tau_{i, k_{i}}} u_{i, j} \widetilde{P}_{i, j-1}\left(\bmod P_{\mathbf{k}}\right), \quad \check{u}^{(\mathbf{k})}, \check{u}_{\mathbf{k}} \in\left[0, P_{\mathbf{k}}\right) .
\end{aligned}
$$

According to (9)-(14), we have $p_{i, \tau_{i, k_{i}}}=p_{i}, k_{i}=1, \ldots, m, i=1, \ldots, s$. By (9), we get $a_{i, \tau_{i, k_{i}}} \equiv \sigma_{i, \tau_{i, k_{i}}}^{-1}(0)-\sigma_{i, \tau_{i, k_{i}}}^{-1}(1) \equiv u_{i, \tau_{i, k_{i}}}^{*}-u_{i, \tau_{i, k_{i}}}\left(\bmod p_{i}\right)$. 
From (16), we obtain $a_{i, \tau_{i, k_{i}}}=\mathfrak{a}_{i}, k_{i}=1, \ldots, m, i=1, \ldots, s$. Hence

$$
\check{u}^{(\mathbf{k})} \equiv \check{u}_{\mathbf{k}}+A_{\mathbf{k}}\left(\bmod P_{\mathbf{k}}\right), \quad \text { where } \quad A_{\mathbf{k}} \equiv \sum_{i=1}^{s} M_{i, \mathbf{k}} P_{\mathbf{k}} p_{i}^{-1} \mathfrak{a}_{i}\left(\bmod P_{\mathbf{k}}\right)
$$

with $A_{\mathbf{k}} \in\left[0, P_{\mathbf{k}}\right)$.

Let $\mathbf{w}=\left(w_{1}, \ldots, w_{s}\right)=H_{\mathcal{P}}^{\Sigma}(n, \mathbf{x})=\widetilde{\Sigma}\left(T_{\mathcal{P}}^{n}(\mathbf{x})\right)$. We see from (21) and (24) that

$$
\begin{aligned}
\mathbf{w} \in B^{(\mathbf{k})} & \Longleftrightarrow \quad w_{i, j}=y_{i, j}, \quad j \in\left[1, \tau_{i, k_{i}}\right), \quad w_{i, \tau_{i, k_{i}}}=0, \quad i \in[1, s] \\
& \Longleftrightarrow \quad \sigma_{i, j}\left(w_{i, j}\right)=u_{i, j}, \quad 1 \leqslant j \leqslant \tau_{i, k_{i}}-1, \\
& \sigma_{i, j}\left(w_{i, \tau_{i, k_{i}}}\right)=u_{i, \tau_{i, k_{i}}}^{*} \quad i=1, \ldots, s \\
& \Longleftrightarrow \quad\left[T_{\mathcal{P}}^{n}(\mathbf{x})\right]_{\tau_{\mathbf{k}}}=\mathbf{u}^{(\mathbf{k})} .
\end{aligned}
$$

Applying (18), (19), (20), (24) and (25), we have

$$
\begin{aligned}
H_{\mathcal{P}}^{\Sigma}(n, \mathbf{x}) \in B^{(\mathbf{k})} & \Longleftrightarrow\left[T_{\mathcal{P}}^{n}(\mathbf{x})\right]_{\tau_{\mathbf{k}}}=\mathbf{u}^{(\mathbf{k})} \\
& \Longleftrightarrow\left[H_{\mathcal{P}}\left(n+W_{\mathbf{m}}(\mathbf{x})\right)\right]_{\tau_{\mathbf{k}}}=\mathbf{u}^{(\mathbf{k})} \\
& \Longleftrightarrow n+W_{\mathbf{m}}(\mathbf{x}) \equiv \check{u}^{(\mathbf{k})}\left(\bmod P_{\mathbf{k}}\right) \\
& \Longleftrightarrow n \equiv v_{m}+A_{\mathbf{k}}\left(\bmod P_{\mathbf{k}}\right),
\end{aligned}
$$

where $v_{m} \equiv-W_{\mathbf{m}}(\mathbf{x})+\check{\mathbf{u}}_{\mathbf{m}} \equiv-W_{\mathbf{m}}(\mathbf{x})+\check{\mathbf{u}}_{\mathbf{k}}\left(\bmod P_{\mathbf{k}}\right)$ and $v_{m} \in\left[0, P_{\mathbf{m}}\right)$.

Hence

$$
H_{\mathcal{P}}^{\Sigma}(n, \mathbf{x}) \in B^{(\mathbf{k})} \Longleftrightarrow n \equiv v_{m}+A_{\mathbf{k}}\left(\bmod P_{\mathbf{k}}\right), \quad v_{m} \in\left[0, P_{\mathbf{m}}\right), n \geqslant 0 .
$$

\subsection{Completion of the proof of Theorem}

Bearing in mind that

$$
\max _{1 \leqslant M \leqslant N} M D^{*}\left(\left(H_{\mathcal{P}}^{\Sigma}(n, \mathbf{x})\right)_{n=1}^{M}\right) \geqslant N^{-1} \sum_{1 \leqslant M \leqslant N} M D^{*}\left(\left(H_{\mathcal{P}}^{\Sigma}(n, \mathbf{x})\right)_{n=1}^{M}\right),
$$

we get that it is sufficient to find the lower bound of the main value of discrepancy function to prove Theorem.

Lemma 1 Let

$$
\alpha_{m}=\frac{1}{P_{\mathbf{m}}} \sum_{M=1}^{P_{\mathbf{m}}} \Delta\left(B_{\mathbf{y}},\left(H_{\mathcal{P}}^{\boldsymbol{\Sigma}}(n, \mathbf{x})\right)_{n=v_{m}}^{v_{m}+M-1}\right) .
$$


Then

$$
\alpha_{m}=\sum_{1 \leqslant k_{1}, \ldots, k_{s} \leqslant m}\left(\frac{1}{2}-\frac{A_{\mathbf{k}}}{P_{\mathbf{k}}}-\frac{1}{2 P_{\mathbf{k}}}\right) .
$$

Proof Let $\mathcal{H}_{n}=H_{\mathcal{P}}^{\Sigma}(n, \mathbf{x})$. Using (26), we have

$$
\sum_{n=v_{m}+M_{1} P_{\mathbf{k}}}^{v_{m}+\left(M_{1}+1\right) P_{\mathbf{k}}-1}\left(\mathbf{1}_{B^{(\mathbf{k})}}\left(\mathcal{H}_{n}\right)-P_{\mathbf{k}}^{-1}\right)=0
$$

and

$$
\begin{aligned}
& \sum_{n=v_{m}+M_{1} P_{\mathbf{k}}}^{v_{m}+M_{1} P_{\mathbf{k}}+M_{2}-1}\left(\mathbf{1}_{B^{(\mathbf{k})}}\left(\mathcal{H}_{n}\right)-P_{\mathbf{k}}^{-1}\right)=\sum_{n \in\left[v_{m}, v_{m}+M_{2}\right)}\left(\mathbf{1}_{B^{(\mathbf{k})}}\left(\mathcal{H}_{n}\right)-P_{\mathbf{k}}^{-1}\right) \\
& =\sum_{\substack{n \in\left[v_{m}, v_{m}+M_{2}\right) \\
n=v_{m}+A_{\mathbf{k}}}} 1-M_{2} P_{\mathbf{k}}^{-1} \\
& =\mathbf{1}_{\left[0, M_{2}\right)}\left(A_{\mathbf{k}}\right)-M_{2} P_{\mathbf{k}}^{-1},
\end{aligned}
$$

with $M_{1} \geqslant 0$ and $M_{2} \in\left[0, P_{\mathbf{k}}\right), M_{1}, M_{2} \in \mathbb{Z}$. From (1) and (22), we get

$$
\begin{array}{r}
\Delta\left(B_{\mathbf{y}},\left(\mathcal{H}_{n}\right)_{n=v_{m}}^{v_{m}+M-1}\right)= \\
\sum_{n=v_{m}}^{v_{m}+M-1}\left(\mathbf{1}_{B_{\mathbf{y}}}\left(\mathcal{H}_{n}\right)-y_{1} \cdots y_{s}\right)=\sum_{k_{1}, \ldots, k_{s}=1}^{m} \rho(\mathbf{k}, M), \\
\text { where } \rho(\mathbf{k}, M)=\sum_{n=v_{m}}^{v_{m}+M-1}\left(\mathbf{1}_{B^{(\mathbf{k})}}\left(\mathcal{H}_{n}\right)-P_{\mathbf{k}}^{-1}\right) .
\end{array}
$$

By (27), we obtain

$$
\alpha_{m}=\sum_{1 \leqslant k_{1}, \ldots, k_{s} \leqslant m} \alpha_{m, \mathbf{k}}, \quad \text { where } \quad \alpha_{m, \mathbf{k}}=\frac{1}{P_{\mathbf{m}}} \sum_{M=1}^{P_{\mathbf{m}}} \rho(\mathbf{k}, M)
$$

Bearing in mind (29)-(30), we derive

$$
\begin{gathered}
\alpha_{m, \mathbf{k}}=\frac{1}{P_{\mathbf{m}}} \sum_{M_{1}=0}^{P_{\mathbf{m}} / P_{\mathbf{k}}-1} \sum_{M_{2}=1}^{P_{\mathbf{k}}}\left(\sum_{n=v_{m}}^{v_{m}+M_{1} P_{\mathbf{k}}-1}\left(\mathbf{1}_{B^{(\mathbf{k})}}\left(\mathcal{H}_{n}\right)-P_{\mathbf{k}}^{-1}\right)\right. \\
\left.+\sum_{n=v_{m}+M_{1} P_{\mathbf{k}}}^{v_{m}+M_{1} P_{\mathbf{k}}+M_{2}-1}\left(\mathbf{1}_{B}(\mathbf{k})\left(\mathcal{H}_{n}\right)-P_{\mathbf{k}}^{-1}\right)\right)
\end{gathered}
$$




$$
\begin{aligned}
& =\frac{1}{P_{\mathbf{m}}} \sum_{M_{1}=0}^{P_{\mathbf{m}} / P_{\mathbf{k}}-1} \sum_{M_{2}=1}^{P_{\mathbf{k}}}\left(\mathbf{1}_{\left[0, M_{2}\right)}\left(A_{\mathbf{k}}\right)-M_{2} P_{\mathbf{k}}^{-1}\right) \\
& =\frac{1}{P_{\mathbf{k}}} \sum_{M_{2}=1}^{P_{\mathbf{k}}}\left(\mathbf{1}_{\left[0, M_{2}\right)}\left(A_{\mathbf{k}}\right)-M_{2} P_{\mathbf{k}}^{-1}\right) \\
& =\frac{P_{\mathbf{k}}-A_{\mathbf{k}}}{P_{\mathbf{k}}}-\frac{P_{\mathbf{k}}\left(P_{\mathbf{k}}+1\right)}{2 P_{\mathbf{k}}^{2}}=\frac{1}{2}-\frac{A_{\mathbf{k}}}{P_{\mathbf{k}}}-\frac{1}{2 P_{\mathbf{k}}} .
\end{aligned}
$$

Using (31), we have

$$
\alpha_{m}=\sum_{1 \leqslant k_{1}, \ldots, k_{s} \leqslant m}\left(\frac{1}{2}-\frac{A_{\mathbf{k}}}{P_{\mathbf{k}}}-\frac{1}{2 P_{\mathbf{k}}}\right) .
$$

\section{Lemma 2 With notations as above,}

$$
\left|\alpha_{m}\right| \geqslant \frac{m^{s}}{4 p_{0}} \quad \text { for } m \geqslant 2 p_{0} .
$$

Proof From (16) and (25), we get

$$
[0,1) \ni \frac{A_{\mathbf{k}}}{P_{\mathbf{k}}} \equiv \sum_{1 \leqslant i \leqslant s} \frac{M_{i, \mathbf{k}} P_{\mathbf{k}} p_{i}^{-1} \mathfrak{a}_{i}}{P_{\mathbf{k}}} \equiv \frac{c_{1} \mathfrak{a}_{1}}{p_{1}}+\cdots+\frac{c_{s} \mathfrak{a}_{s}}{p_{s}}(\bmod 1) .
$$

Applying (17) and (28), we derive

$$
\alpha_{m}=m^{s}\left(\frac{1}{2}-\{\alpha\}\right)-\sum_{1 \leqslant k_{1}, \ldots, k_{s} \leqslant m} \frac{1}{2 P_{\mathbf{k}}}, \quad \text { where } \alpha=\frac{d_{1}}{\widehat{p}_{1}}+\cdots+\frac{d_{s}}{\widehat{p}_{s}},
$$

$\left(d_{i}, \widehat{p}_{i}\right)=1, \widehat{p}_{i}>1, i=1, \ldots, s$, and $\{x\}$ is the fractional part of $x$. We have that if $\widehat{p}_{0}=\widehat{p}_{1} \widehat{p}_{2} \cdots \widehat{p}_{s} \not \equiv 0(\bmod 2)$ then $\alpha \neq \equiv 1 / 2(\bmod 1)$. Let $\widehat{p}_{v} \equiv 0(\bmod 2)$ for some $v \in[1, s]$, and let $\alpha \equiv 1 / 2(\bmod 1)$. Then

$$
\frac{\widehat{p}_{\nu} / 2-d_{v}}{p_{v}} \equiv \sum_{\substack{1 \leqslant i \leqslant s \\ i \neq v}} \frac{d_{i}}{\widehat{p}_{i}}(\bmod 1), \quad a_{1} \equiv a_{2}\left(\bmod p_{0}\right),
$$

with $a_{1}=\widehat{p}_{0}\left(\widehat{p}_{v} / 2-d_{v}\right) / \widehat{p}_{v}$ and $a_{2}=\sum_{i \neq v} \widehat{p}_{0} d_{i} / \widehat{p}_{i}$. Let $j \in[1, s]$ and $j \neq v$. We see that $a_{1} \equiv 0\left(\bmod \widehat{p}_{j}\right)$ and $a_{2} \not \equiv 0\left(\bmod \widehat{p}_{j}\right)$. We get a contradiction. Hence $\alpha \neq 1 / 2(\bmod 1)$. We have

$$
\left|\frac{1}{2}-\{\alpha\}\right|=\left|\frac{1}{2}-\left\{\frac{d_{1}}{\widehat{p}_{1}}+\cdots+\frac{d_{s}}{\widehat{p}_{s}}\right\}\right|=\frac{|a|}{2 \widehat{p}_{0}} \quad \text { for some integer } a .
$$

Thus $|1 / 2-\{\alpha\}| \geqslant 1 /\left(2 \widehat{p}_{0}\right) \geqslant 1 /\left(2 p_{0}\right)$ with $p_{0}=p_{1} \cdots p_{s},\left(p_{0}, \widehat{p}_{0}\right)=\widehat{p}_{0}$. 
Bearing in mind that $P_{\mathbf{k}} \geqslant 2^{k_{1}+k_{2}+\cdots+k_{s}}$, we obtain from (32) that

$$
\left|\alpha_{m}\right| \geqslant \frac{m^{s}}{2 p_{0}}-\frac{1}{2}=\frac{m^{s}}{2 p_{0}}\left(1-\frac{p_{0}}{m^{s}}\right) \geqslant \frac{m^{s}}{4 p_{0}} \quad \text { for } \quad m \geqslant 2 p_{0} .
$$

This completes the proof.

Going back to the proof of Theorem, by (7) and (13), we get

$$
\begin{aligned}
m^{s}\left(4 p_{0}\right)^{-1} & \geqslant\left(4 p_{0}\right)^{-1} C_{1}^{-s} \log _{2}^{s} N \geqslant 2 C^{-1} \log _{2}^{s} N, \\
m & \geqslant C_{1}^{-1} \log _{2} N \geqslant 2 p_{0},
\end{aligned}
$$

where $C_{1}=s q_{0}^{s+1} \log _{2} q_{0}, C=8 q_{0}^{s} C_{1}^{s}$ and $q_{0}^{s} \geqslant p_{0}$.

Using (15) and (26), we have that $v_{m}+P_{\boldsymbol{\tau} m} \leqslant 2 P_{\mathbf{m}} \leqslant N$. According to (33), (27) and (2), we obtain

$$
\begin{aligned}
2 C^{-1} \log _{2}^{s} N & \leqslant m^{s}\left(4 p_{0}\right)^{-1} \leqslant\left|\alpha_{m}\right| \leqslant \max _{1 \leqslant M \leqslant P_{\mathbf{m}}} M D^{*}\left(\left(H_{\mathcal{P}}^{\Sigma}(n, \mathbf{x})\right)_{n=v_{m}}^{v_{m}+M-1}\right) \\
& \leqslant \max _{1 \leqslant L, L+M \leqslant 2 P_{\mathbf{m}}} M D^{*}\left(\left(H_{\mathcal{P}}^{\Sigma}(n, \mathbf{x})\right)_{n=L}^{L+M-1}\right) \\
& \leqslant 2 \max _{1 \leqslant M \leqslant N} M D^{*}\left(\left(H_{\mathcal{P}}^{\Sigma}(n, \mathbf{x})\right)_{n=1}^{M}\right)
\end{aligned}
$$

Hence Theorem is proved.

Acknowledgments The author is very grateful to the referee for corrections and suggestions which improved this paper.

\section{References}

1. Beck, J., Chen, W.W.L.: Irregularities of Distribution. Cambridge Tracts in Mathematics, vol. 89. Cambridge University Press, Cambridge (1987)

2. Bilyk, D.: On Roth's orthogonal function method in discrepancy theory. Unif. Distrib. Theory 6(1), 143-184 (2011)

3. Faure, H.: Discrépances de suites associées à un système de numération (en dimension un). Bull. Soc. Math. France 109(2), 143-182 (1981)

4. Faure, H., Chaix, H.: Minoration de discrépance en dimension deux. Acta Arith. 76(2), 149-164 (1996)

5. Faure, H., Kritzer, P., Pillichshammer, F.: From van der Corput to modern constructions of sequences for quasi-Monte Carlo rules. Indag. Math. (N.S.) 26(5), 760-822 (2015)

6. Halton, J.H.: On the efficiency of certain quasi-random sequences of points in evaluating multidimensional integrals. Numer. Math. 2, 84-90 (1960)

7. Hellekalek, P.: Regularities in the distribution of special sequences. J. Number Theory 18(1), 41-55 (1984)

8. Levin, M.B.: On the lower bound in the lattice point remainder problem for a parallelepiped. Discrete Comput. Geom. 54(4), 826-870 (2015)

9. Levin, M.B.: On the lower bound of the discrepancy of Halton's sequences: I. C. R. Math. Acad. Sci. Paris Sér. I Math. (to appear)

10. Levin, M.B.: On the lower bound of the discrepancy of $(t, s)$ sequences: II (2015). arXiv:1505.04975v2 
11. Niederreiter, H.: Random Number Generation and Quasi-Monte Carlo Methods. CBMS-NSF Regional Conference Series in Applied Mathematics, vol. 63. Society for Industrial and Applied Mathematics (SIAM), Philadelphia (1992)

12. van der Corput, J.G.: Verteilungsfunktionen I-II. Proceedings. Akadamie van Wetenschappen Amsterdam 38, 813-821, 1058-1066 (1935) 